\title{
De la escena a las aulas: los artistas y la incorporación de la danza española y el baile flamenco a las enseñanzas generales From the stage to the classrooms: artists and the incorporation of Spanish and flamenco dance into the general education \\ *Patricia Bonnin-Arias, *EstelaAlarcón Rodríguez, **Ana Colomer-Sánchez \\ *U niversidad Rey Juan Carlos (España), **Universidad N ebrija (España)
}

\begin{abstract}
Resumen. La problemática social del siglo XXI, caracterizado por laincertidumbre y el cambio acelerado, obligan ala escuela a reconfigurarse. Las artes se presentan como una oportunidad para formar ciudadanos capaces de afrontar estos retos, ofreciendo competencias en materia de creatividad, identidad y sentido de comunidad, muy ligadas a las artes autóctonas. Sin embargo, lasventajas derivadas del aprendizaje de la danza españolay el bail e flamenco, parecen estar siendo desaprovechadas por las enseñanzas generales en España. Este trabajo pretende indagar en el papel que juegan los profesionales del mundo de la danza autóctona en la educación obligatoria en España, explorando en las configuraciones perceptivas y autoperceptivas de los artistas ante la incorporación de la misma a la escuela. Se ha efectuado un estudio descriptivo no probabilístico, mediante cuestionar ios semiestructuradas que recogen la experiencia en primera persona y la visión de artistas reputados en el ámbito del objeto. Los resultados inciden en la orientación general del diseño e implementación de los estudios de danza en la escuela, sus beneficios para afrontar los retos sociales actual es, las etapas educativas oportunas para el calado, la afinidad con otros docentes y asignaturas, infraestructuras y la val oración de lacual ificación de los profesores que imparten danzaen la educación general, brindando una aproximación al rol presente y futuro de estos artistas en la escuela.
\end{abstract}

Palabras clave: Pedagogía; Arte; Currículo; Docente; Identidad profesional.

\begin{abstract}
The demand for finding solutions to the social challenges of the 21st century, characterized by uncertainty and rapid change, requires a reconfiguration of the school system. In this context, the arts can offer an opportunity to prepare citizens in a better way to face new challenges. Arts provide valuable competencies like creativity, identity and the creation of a sense of community, which can be especially beneficial when they derive from the autochthonous culture. However, the advantages related to learning Spanish and flamenco dance seem to be neglected by the general school system in Spain. The present study explores the perceptive and self-perceptive configurations of artists about the incorporation of autochthonous dance in school. A descriptive and non-probabilistic analysis has been performed by means of a semi-structured survey. The survey focused on first-person experience and the vision of reputed artists in the fields of study. The results shed light upon the general orientation for the design and implementation of autochthonous dance classes in school, the benefit of their inclusion in the curriculum for the community and for facing the challenges of the 21st century. M oreover, they indicate which are the most convenient stages of education for the introduction of the subject in order to ensure affinity with other teachers and their disciplines, as well as with the available infrastructure. Lastly, the results highlight the importance of the qual ifications of the current dance teachers in the education system and suggest the present and future roles of dance artists in schools.
\end{abstract}

Key words: Pedagogy; Art; Syllabus; Teacher; Professional Identity.

\section{Introducción}

La danza autóctona es una de las manifestaciones artísticas significativas en la articulación de la arquitectura imaginada de los pueblos (Anderson, 1993). Su importancia como elemento vertebrador del sentido de comunidad, su valor como patrimonio cultural y susbeneficios cardinales para afrontar los retos sociales del siglo XXI, hacen que se incorpore al debate educativo.

Fecha recepción: 17-09-20. Fecha de aceptación: 22-11-20

Ana Colomer Sánchez

ana.colomer.universidad@gmail.com
Loscolectivosinvolucrados en el binomio danza-educación justifican su vinculación mediante un enfoque esencial ista - Art for Art's sake- que defiende la experiencia en las artes como única, diferenciada y responsable de la transmisión de valores humanizadores que nos permiten vivir en comunidad (Eisner, 2008; Zakaras \& Lowell, 2004). Además, la formación genuina en programas de educación dancística permite a los estudiantes aprender complejas secuencias danzadas, desarroIlar habilidades de creación y ejecución del movimiento, fomentar el relacionamiento visual con el público, colaborar con el compromiso y el entusiasmo en el aprendizaje y brindar la oportunidad de trabajar con 
profesionales de la danza (Horowitz, 2003).

Por otro lado, la corriente contextualista se centra en su relación con las asignaturas del currículo escolar mediante la aplicación transversal y metodológica en las diversas materias (Touriñán, 2011; U nesco, 2006). W inner, Goldstein y Vincent-Lancrin (2014) consideran que todavía faltan estudios que aporten evidencia científica concluyente acerca de la incidencia de la danza sobre habilidades académicas tales como la lectoescritura, las matemáticas o el razonamiento espacial; sin embargo, los autores subrayan los efectos positivos en una variedad de pruebas cognitivas, halla dos en los participantes de un programa de educación dancística no orientado específicamente a apoyar resultados académicos, los cuales han sido atribuidos al aumento de la motivación, concentración y disciplina derivados de su práctica, y esto parece extenderse hacia otras materias académicas. Más allá de la transferencia cognitiva, los resultados académicos o las habilidades motoras cuantificables, la danza y sus experiencias holísticas, catalizan cambios no impuestos y constituyen un aprendizaje de alta calidad que, necesariamente, influye en el sentido de comunidad, al plantear el estar juntos, el vivir juntos, la aceptación de uno mismo, la colaboración con los demás y el fomento de la creatividad (Anttila \& Svendle, 2019).

Por su parte, la incertidumbre, aceleramiento del cambio y la exigencia de encontrar soluciones a la problemática inherente al siglo XXI, convierte en imperativo el fomento de las competencias creativas desde la escuela (Craft, 2005, 2011; Klimenko, 2008). Si bien la creatividad no es un rasgo exclusivo del arte (Gardner, 2011), su evidente asociación ha fortalecido el discurso de la introducción de las artes en el currículo escolar como parte de la formación de los ciudadanos del futuro (Runco, 1989; W inner, Goldstein \&Vincent-Lancrin, 2014), teoría avalada por su capacidad de desarrollar la originalidad, lafluidez y laflexibilidad (H efferon \& O llis, 2006). En lo que respecta a la danza, la aplicación del movimiento en las aulas hace prosperar la capacidad neuronal del aprendizaje cinestésico, acrecentando la memorización y el aprendizaje mediante el equilibrio de los dos hemisferios cerebrales; el derecho el de la imaginación y el izquierdo el de la razón, mejorando el rendimiento académico (M éndez-Giménez, 2020).

La danza podrá ser un mecanismo positivo para potenciar la creatividad, siempre que se implemente en la escuela de manera abierta y sin estructuras predeterminadas que coarten la libertad del alumno (Laban, 1978). Sin embargo, aunque en algunos contextos las luchas de la educación dancística por ganarse un lugar en la educación obligatoria han dado sus frutos, preocupa que una deficiente implementación subvierta su ca rácter de práctica creativa, debido a diversos factores, entre ellos, la presión por el rendimiento académico 0 la mejorable formación en danza de los docentes (Ackroyd, 2001; 0 fsted, 2006). Para paliar este déficit, Chappell, Rolfe, Jobbinsy Craft (2011) ponen el acento en las asociaciones creativas entre maestros y artistas experimentados, como prácticas altamente innovadoras y claves en el proceso de humanización del discente, que alientan la empatía, la propiedad compartida y el sentido de identidad grupal.

0 tro eje del debate inherente a la educación dancística en la escuela tiene que ver con la técnica a implementar en contextosno profesionalizadores. Desde la formulación del concepto de Danza Educativa, Laban (1978) conmina a no centrarse en la ejecución vir tuosa, sino en el efecto benéfico sobre el alumnado de una danza neutra, carente de modelos de imitación, que respete las etapas del desarrollo del educando y se centre más en los procesos que en los resultados. En coherencia con esta línea filosófica, diversos autores que han promovido esquemas de trabajo desprovistos de una estetización específica, de una gestualidad convenida 0 de un vocabulario estrictamente academizado, sí se han mostrado interesados en las danzas autóctonas, por tra tarse de una vía de acceso a la cultura y a las vivencias de la comunidad (Hasselbach, 1979; Robinson, 1999).

\section{U n espacio para la danza en el currículo esco- lar español}

En España, la danza está buscando su encaje en un currículo que delata el todavía exiguo compromiso social y el desinterés de la clase política para con este arte (Matos-Duarte, Smith \& Muñoz, 2020; Rubio, 2016). Se puede rastrear la evolución de la presencia de la danza con fines no profesional izadores en el ámbito formal en las leyes orgánicas de educación a través de las asignaturas de Educación Física, Artística y Música, desde la implantación de la LO GSE en 1990 (De las Heras \& Calderón, 2018). Su ya cuestionable protagonismo, basado en la jerarquización de currículo, ha ido mermando hasta quedar marginada en asignaturas de carácter optativo, exceptuando Educación Física(O rtiz, 2015). El hecho deno haber adquirido una entidad propiacomo asignatura, sumado a la insuficiente formación en danza del profesorado que la imparte, la carencia de un currículo especializado, la escasez de medios y materiales de consulta, los déficits de infraestructura y los estereoti- 
pos de género, son todos ellos indicadores de su limita da prevalencia en el sistema educativo (Algar \& Algar, 2015; M atos-Duarte, Smith \& M uñoz, 2020; O rtiz, 2015; Peña de H ornos \& Vicente, 2019).

O rtiz (2015) ofrece un análisis de la presencia de la danza en la educación obligatoria y los docentes vincula dos a ella. Todavía vigente la LO M CE, estas enseñanzas se vertebran en Educación Primaria a través de Educa ción M usical, una asignatura que ha pasado a ser optativa y que suele ser impartida por especialistas en M úsica o con mención en esta disciplina. Respecto a la Educación Secundaria, la danza aparece en Educación Física del primer ciclo, relacionada con la producción de acciones motrices con finalidad expresiva, aś como en segundo ciclo, centrada en la composición a partir de la selección y ajustes de elementos para conseguir dicha motricidad expresiva. Dichos contenidos son impartidos por licenciadosy graduados en Educación Física. Por otra parte, dentro del bloque de asignaturas específicas que se ofertan para segundo ciclo de Secundaria se recoge, además, la asignatura de Artes Escénicas y Danza, en cuyo primer y tercer bloque se abordan contenidos relacionados con la danza, que se suman a los bloques dedicados al arte dramático y a otras artes escénicas. Cabe preguntarse qué docentes y con qué preparación deberán enfrentarse a tal variedad de contenidos ( 0 rtiz, 2015).

La transferencia de competencias educativas a las comunidades autónomas podría explicar el heterogé neo calado de la danza española y el baile flamenco en los planes de estudio. El análisis de la presencia de la danza en la asignatura de Educación Física de los currículos autonómicos realizado por López, López y Vicente (2020), ofrece una visión panorámica de la situación desde una perspectiva regional. Considerándose la Educación Física un área clave para incidir en la competencia intercultural (M atos-Duarte, Smith \& Muñoz, 2020), mientras el $83.3 \%$ de los currículos autonómicos referencian expresamente las danzas propias de su región en esta asignatura, sólo un $11.1 \%$ mencionan las danzas de otras regiones de España, contrastando con las tres cuartas partes que señalan la relevancia de las «danzas del mundo» o de otros contextos culturales, frecuentemente internacionales (López, López \&Vicente, 2020).

Parece constatarse una tendencia más favorable al baile flamenco, en detrimento de las demás modalida des de la danza española cuya presencia en las aulas es residual (Algar \& Algar, 2015). Andalucía es la única autonomía que ha introducido el flamenco de forma expresa en la educación (De las Heras \& García, 2019) con una intencionalidad de reivindicación patrimonial, de identidad y por su carácter multicultural e inclusivo, que permite allanar las diferencias social es entre culturas hegemónicas y fronterizas (Gutiérrez, 2010; López, 2004; Padial-Ruz, Ibáñez-Granados, Fernández \& U bagoJiménez, 2019; Rodríguez-Q uiles, 2006). Esto contrasta con otras regiones españolas en las que el (anti)flamenquismo ha sido instrumentalizado para articular un discurso identitario definido por un supuesto opuesto constitutivo (Holguín, 2013), en un momento en el que el flamenco ha llegado a considerarse parte del patrimonio universal y está obteniendo una amplia difusión en una sociedad globalizada (U nesco, 2010).

Cabe subrayar, además, que el concepto de danza española institucionalizado en los conser vatorios de danza dependientes de todas las comunidades autónomas responden al modelo propuesto por M ariemma (1997), en el cual, además del baile flamenco, convergen la escuela bolera, la danza estilizada y la danza tradicional, la cual abarca en toda su amplitud las manifestaciones dancísticas regionales españolas. Pese a las ventajas e inconvenientesque puedaplantear dicho modelo (López, 2018), este amplio espectro dancístico ofrece numerosas opciones asociadas a la identidad cultural de una 0 más regiones, además de mostrarse como una vía de fraternal acercamiento a otras realidades culturales que se manifiestan dentro de las fronteras estatales, que no por ser diversas deben resultar antagónicas, al menos no en un contexto educativo.

En otro orden de cosas, las frecuentes trabas burocráticas de las administraciones para ofrecer los espacios escénicos para los proyectos contrastan con la predisposición de artistas para colaborar con los profesores y centros educativos involucrados (Algar \& Algar, 2015; Jiménez, 2017). En cuanto a los discentes, si bien inicialmente tienden a ridiculizar e infravalorar estas experiencias, parecen experimentar posteriormente una transformación que les conmina a involucrarse activamente, posibilitando la transmisión de valores culturales y patrimonial es a través de la danza autóctona (Díaz, 2012).

\section{El profesorado de danza en las aulas de educa- ción obligatoria}

Partiendo del axioma de que la calidad de un sistema educativo no puede ser superior a la de sus docentes, ponemos el foco en el profesorado como bisagra entre el marco regulador y el educando. Los delineamientos de calidad involucran a los profesores 
generalistas, profesores de asignaturas artísticas y a los artistas, en un trabajo colaborativo dentro de su ámbito competencial (Unesco, 2006), que genera un modelo cuádruple de relación que incluye al maestro como artista, al artista como educador, el arte de la enseñanza y el trabajo artístico como modelo y educador (Chappell, Rolfe, Jobbins \& Craft, 2011). Por su parte, Burnard, Holliday, Jasilek y Nikolova (2018) insisten en que la presencia del artista en las aulas contribuye a la interrupción de la monotonía de la vida ordinaria, generando un espacio heterotópico donde el cambio y la transformación son posibles.

Cabe, además, examinar el proceso de migración del artista del escenario a la escuela, dos ámbitos con diferenciadas configuraciones culturales (Day, 1986; Hall, 2010). Lejos de traducirse en una dicotomía, los sujetos pueden funcionar de forma dual, compatibilizando su práctica docente con la artística (Thornton, 2005) mediante un proceso en el que construyen y renegocian sus identidades y lealtades profesionales como una experiencia transformadora, que, aunque no esté exenta de tensiones (Hall, 2010), los hace significativamente más efectivos en su labor educativa (NSEAD, s.f.).

La formación didáctica de los artistas españoles suele ser aneja a las enseñanzas artísticas superiores y universitarias de arte. En el caso de la danza, se canaliza a través de los títulos superiores de danza ofertados por los diversos conservatorios superiores de danza y centros autorizados por las comunidades autónomas, así como de losgrados universitariosen Ciencias de la Danza (Universidad Europea de Madrid), Pedagogía en Artes Visuales y Danza (Universidad Rey J uan Carlos), Artes Visuales y Danza - M enciones (Universidad Rey Juan Carlos) y el grado en Danza (U niversidad Católica de Murcia), aunque las dos primeras titulaciones ya están extintas. En ellos, las competencias didácticas suelen estar orientadas hacia perfiles profesionalizadores. Sin embargo, también se ofrecen asignaturas específicas e itinerarios formativos globalmente orientados a la danza educativa y comunitaria, y, por tanto, a la danza ama teur, algunos todavía pendientes de implantación (Conservatorio Superior de Danza «María de Ávila», s.f.). Tras la obtención de estas titulaciones, salvo excepciones, es necesario realizar la formación de posgrado habilitante para la docencia en Secundaria.

Pese a que los artistas-docentes egresados de las titulaciones superiores y universitarias de danza ya se han insertado en el mercado laboral, salvo excepciones, la formación en danza en la educación general continúa en manos de profesores de otras asignaturas, arrinco- nando a los egresados especializados en el ámbito formal (profesionalizador), no formal e informal. Diversos estudios cuestionan la formación en danza del profesorado que la imparte en los colegios e institutos, considerándola reducida e inespecífica, lo cual afecta a su impartición (García, 2002; Troya \& Cuéllar, 2013) y produce un desbalance entre el peso de la danza en el currículo y su cualificación (Cuéllar \& Pestano, 2013).

Todo lo expuesto nos lleva al objetivo principal de esta investigación: conocer el papel que juegan los profesionales del mundo de la danza española y el baile flamenco en la educación obligatoria en España, para recoger desde su experiencia en primera persona, su implicación pasada y presente como docentes y las futuras maneras de participación en la enseñanza general.

Específicamente se pretende:

Indagar en la visión de los artistas respecto a la inclusión y a la situación actual de la danza española y el baile flamenco en el sistema educativo español.

- Explorar las implicaciones de la enseñanza de danza española y flamenco en la escuela respecto al sentido de pertenencia al grupo e identidad cultural desde la perspectiva de los artistas.

Determinar el rol actual de los artistas en los diferentes niveles educativos y asignaturas obligatorias, optativas y de libre elección.

Valorar si las infraestructuras de la escuela y las actividades culturales como espectáculos y artistas son viables para una educación de calidad.

\section{Método}

El presente estudio se define como descriptivo observacional siguiendo una metodología cuantitativa. En cuanto a la selección de la muestra se realizó mediante un muestro no probabilístico por conveniencia. Los participantes representan a un subgrupo compuesto por sujetos elegidos específicamente. Por consiguiente, los mismos se seleccionaron siguiendo los siguientes criterios de inclusión: (i) adscripción a un centro de trabajo referente nacional o mundial dentro del ámbito de la danza española y el baile flamenco; (ii) participación reciente en giras nacionales e internacionales de danza española y baile flamenco; (iii) obtención de al gún premio o reconocimiento de la crítica o de las instituciones públicas y culturales; (iv) dirección de una compañía de danza, todo ello en los últimos diez años.

\section{Participantes}

La muestra fue recogida de enero a marzo de 2020 
a nivel estatal en compañías e instituciones referenciales de este ámbito. Inicialmente participaron 69 profesionales de la danza española y el baile flamenco. Sin embargo, tras un proceso de revisión realizado por dos expertas de danza con prolongada trayectoria artística profesional y un nivel universitario de estudios, fueron descartados ocho participantes que no cumplían con los requisitos de inclusión. Así, la muestra estuvo formada por un total de 61 profesionales de la danza española y baile flamenco incluidos dentro de las categorías de bailarines/ bailaores (88.5 \%) y cantantes/ músicos (11.5 $\%)$, dotados de una reconocida trayectoria profesional en el panorama internacional y nacional, a los que se puede considerar, por tanto, informantes al tamente cua lificados y representativos del patrimonio cultural. En cuanto a los parámetros de inclusión el $90.2 \%$ de los participantes considera que sus lugares de trabajo constituyen un referente cultural, el $95.1 \%$ ha participado en giras nacionales y un $91.8 \%$ en giras internaciona les.

Entre los lugares de trabajo encontramos emblemáticas organizaciones artísticas, tales como el Ballet Nacional de España, el tablao flamenco «Corral dela M orería» y las compañías vinculadasa O IgaPericet, Marco Flores, Antonio Gades, Eduardo Guerrero, Sara Baras, María Pagés, Daniel Doña, Antonio Márquez, Pedro Embrujo, Rafael Aguilar, Paco Peña y Andoitz Ruibal entre otras personalidades cuyas identidades se preservan.

En cuanto a la distribución de los participantes, el $54.1 \%$ eran mujeres y el $45.9 \%$, hombres. El rango de edad entre los 30 y los 44 años fue el de mayor presencia ( $54.1 \%$ de la muestra). Dentro del papel del artista, seles pidió queindicasen su profesión principal, siendo el $6.6 \%$ creadores, el $63.9 \%$ intérpretes y un $29.5 \%$

\begin{tabular}{|c|c|c|c|}
\hline & & Frecuencia & Porcentaje \\
\hline \multirow{2}{*}{ Sexo } & Mujeres & 33 & 54.1 \\
\hline & Hombres & 28 & 45.9 \\
\hline \multirow{4}{*}{ Edad, años } & $18-29$ & 15 & 24.6 \\
\hline & $30-44$ & 33 & 54.1 \\
\hline & $45-64$ & 8 & 13.1 \\
\hline & En blanco & 5 & 8.2 \\
\hline \multirow{2}{*}{ Papel del artista } & Bailarín/ bailaor & 35 & 88.5 \\
\hline & Músico & 7 & 11.5 \\
\hline \multirow{5}{*}{$\begin{array}{l}\text { Nivel estudios } \\
\text { artísticos total muestra }\end{array}$} & Elemental & 1 & 1.6 \\
\hline & Autodidacta & 6 & 9.8 \\
\hline & Medio & 16 & 26.2 \\
\hline & Superior & 36 & 59 \\
\hline & Máster & 2 & 3.3 \\
\hline \multirow{2}{*}{ Estudios músicos } & Autodidacta & 5 & 71.4 \\
\hline & Superior & 2 & 28.6 \\
\hline \multirow{5}{*}{ Estudios danza } & Autodidacta & 1 & 1.9 \\
\hline & Elemental & 1 & 1.9 \\
\hline & Medio & 16 & 29.6 \\
\hline & Superior & 34 & 63 \\
\hline & Máster & 2 & 3.7 \\
\hline \multirow{3}{*}{ Profesión principal } & Creador & 4 & $6.6 \%$ \\
\hline & Intérprete & 39 & $63.9 \%$ \\
\hline & Pedagogo & 18 & $29.5 \%$ \\
\hline Total & & 61 & $100 \%$ \\
\hline
\end{tabular}

pedagogos. El $59 \%$ de la muestra tenía estudios superiores y un $9.8 \%$ era autodidacta. Se aprecia que un $71.4 \%$ de los músicos era autodidacta, frente a un 1.9 $\%$ de bailarines / bailaores autodidactas.

\section{Instrumento}

La información fue recogida mediante un cuestionario semiestructurado (Alvira, 2011) preparado por los autores para indagar en las variables sociodemográficas, a fin de adquirir tanto información personal, como la percepción del rol de los artistas de la danza española y el baile flamenco en la enseñanza general.

Dicho cuestionario se dividió en seis epígrafes orientados a responder a la premisa principal y a los objetivos específicos, partiendo del corpus teórico relacionado con el objeto. En el bloque cero se hace referencia a los datos personales y los factores de inclusión; el bloque uno aborda la inclusión de la danza española y el baile flamenco en el sistema educativo español y la participación por parte de los artistas (Grande, 2005; Gutiérrez, 2010; López, 2004; Salazar, 2018; Utrilla, 2007), el bloque dosindaga en el papel de los artistas en la educación general y su posible aportación según el nivel educativo y el tipo de asignatura (Salazar, 2018; M uñoz, 1996; Trigo, Rodríguez \& Moreno, 2019). Por su parte, el bloque tres pretende evaluar la viabilidad de las infraestructuras de la escuela y equipamientos culturales para las actividades pedagógicas y artísticas, para observar si son factibles dentro de la educación general (Giménez, 2012), y ya en los bloques cuatro y cinco se incide en la situación actual de la implantación de la danza dentro de la enseñanza general y el papel del artistaal respecto (De Las Heras \& Calderón, 2018; Gutiérrez, 2010).

Se elaboró un pretest (Alvira, 2011) que fue evalua do por un conjunto de expertos en la materia, todos ellos con estudios superiores, expertos en danza e investigadores, para que las preguntas tuvieran unos parámetros correctos. Se les planteó que atendieran a los objetivos del estudio, claridad del lenguaje, duración del cuestionario, objetividad de las preguntas, entre otras cuestiones. Una vez revisado, se añadieron las consideraciones de los expertos a nivel gramatical, se reordenaron las preguntas y se incorporaron categorías sugeridas. Finalizada la revisión de las objeciones e implementadas las modificaciones recomendadas, se procedió a enviar el cuestionario a los participantes.

\section{Análisis de datos}

Una vez recogidos los datos, se realizó un análisis 
estadístico descriptivo, mediante el programa SPSS para W indows. Para obtener las frecuencias y los porcentajes del conjunto de artistas.

\section{Procedimiento}

La participación en el estudio fue voluntaria y los participantes fueron informados en todo momento del propósito del estudio y del anonimato de sus respuestas. Los cuestionarios se confeccionaron y difundieron a tra vés de una plataforma web (Google Forms) disponible en el siguiente enlace y se completaron individualmente en línea. Cada uno de los participantes estuvo en contacto de manera telefónica 0 en línea con algún responsable del estudio. Para controlar de manera más exhaustiva el cumplimiento de estos requisitos de inclusión, se planteó una batería de preguntas introductorias con vistas a validar la trayectoria profesional de los artistas, asegurando mediante esto la máxima calidad de los registros.

\section{Resultados}

\section{Inclusión de las artes en el sistema educativo español}

Los cuestionarios reflejaron una visión conjunta de los profesionales de danza española y el baile flamenco, así como la participación previa y la voluntad de participar en un proyecto en educación general. De la primera pregunta se desprende que un $52.5 \%$ de los artistas había participado de forma activa en un proyecto de danza en la escuela, frente a un $45.9 \%$ que no 10 había hecho. La pregunta dos expone que al $86.9 \%$ le gustaría participar en proyectos educativos frente a un $13.1 \%$ que no lo tenía claro («tal vez»). De la pregunta cinco se deriva que un $57.4 \%$ desconoce proyectos 0

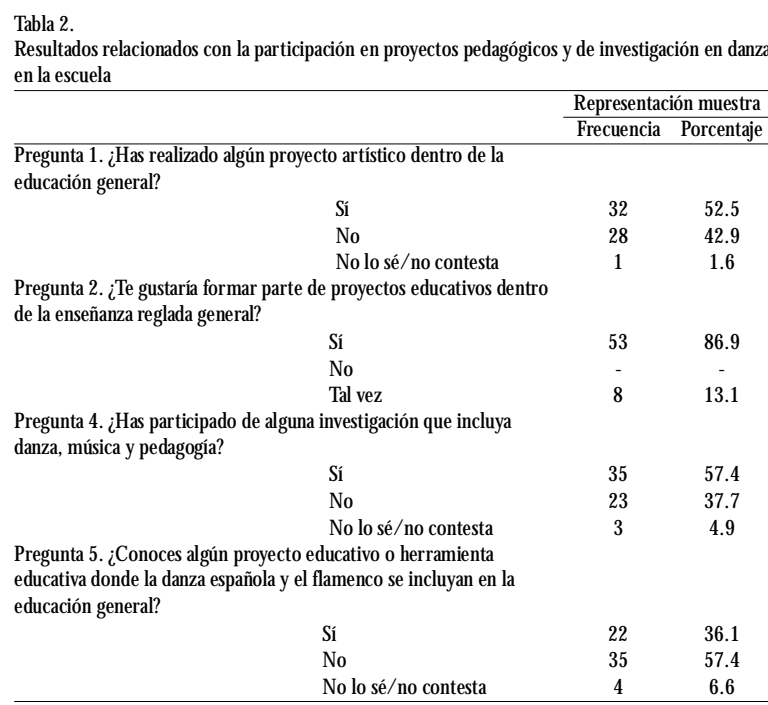

herramientas educativas implementadas en la educación general, en concordancia con las respuestas a la primera pregunta, polarizadas en dos mitades.

Tras indagar en la percepción del impacto de los proyectos educativos en la pregunta tres, un $96.7 \%$ de los encuestados contesta que los proyectos de danza española y baile flamenco dentro de la educación general pueden ser productivos para docentes y discentes. En las respuestas a la pregunta siete se aprecia que más de la mitad de los participantes, un $65.6 \%$, piensa que la danzafavoreceríaformar ciudadanos comprometidoscon las artes y con el patrimonio cultural. En cuanto, a la opinión de los profesionales sobre la incidencia de la danza en el sentido de pertenencia al grupo e identidad (pregunta ocho), el $88.5 \%$ opinó que lo fomentaba, frente a solo un $1.6 \%$ que apreciaba lo contrario.

\begin{tabular}{|c|c|c|}
\hline & \multicolumn{2}{|c|}{ Representación muestra } \\
\hline & Frecuencia & Porcentaje \\
\hline \multicolumn{3}{|l|}{$\begin{array}{l}\text { Pregunta 3. ¿Q ué percepción tienes del impacto o utilidad de un } \\
\text { proyecto educativo que enseñe a la sociedad lo que es el arte } \\
\text { flamenco? }\end{array}$} \\
\hline Poco productivo para el docente y el educando & 1 & 1.6 \\
\hline Productivo para el docente y el educando & 59 & 96.7 \\
\hline No lo sél no contesta & 1 & 1.6 \\
\hline \multicolumn{3}{|l|}{$\begin{array}{l}\text { Pregunta 7. ¿En qué ámbito repercutiría más la enseñanza de la danza } \\
\text { española y el baile flamenco en la escuela? }\end{array}$} \\
\hline $\begin{array}{l}\text { En la formación de ciudadanos comprometidos con las artes } \\
\text { y que val oran su patrimonio. }\end{array}$ & 40 & 65.6 \\
\hline En la formación de nuevo público para la danza & 13 & 21.3 \\
\hline En la formación de nuevos profesionales de danza & 7 & 11.5 \\
\hline No lo sé/ no contesta & 1 & 1.6 \\
\hline \multicolumn{3}{|l|}{$\begin{array}{l}\text { Pregunta 8. ¿C rees que la enseñanza de la danza española y el baile } \\
\text { flamenco en la escuela puede contribuir a reforzar el sentido de } \\
\text { pertenencia al grupo y de identidad en las comunidades en las que se } \\
\text { enseña? }\end{array}$} \\
\hline Sí & 54 & 88.5 \\
\hline No & 1 & 1.6 \\
\hline Tal vez & 6 & 9.8 \\
\hline
\end{tabular}

Papel de los artistas en las leyes educativas dentro de los diferentes niveles educativos y asignaturas: obligatorias, optativas y de libre elección

Preguntados sobre dónde podrían aportar sus conocimientos dentro de la enseñanza general, se aprecia que el $45.9 \%$, casi la mitad de ellos, coinciden que sería en la Educación Primaria, seguido de un $24.6 \%$ que lo haría en la universidad (pregunta nueve). Dentro de la Educación Primaria, el $32.8 \%$ de los participantes cree que participaría en un proyecto relacionado con Lengua Castellana y Literatura, siendo la asigna tura de M atemáticas con un 8.2 \% lade menor puntación (pregunta 10).

En Educación Secundaria, los participantes anteponen la asignatura de Geografía e Historia, un 45.9 \% por delante de la de Lengua Castellana y Literatura $34.4 \%$ - y en último lugar seleccionan las asignaturas de Primera Lengua Extranjera y Matemáticas, tan solo un $4.9 \%$ (pregunta 11). Cabe destacar que, dentro de 
lasasignaturas deSecundaria, tanto Educación Física(60.7 $\%)$, como Música ( $26.2 \%$ ) serían las asignaturas más afines para colaborar con ar tistas de la danza española y baile flamenco.

En el marco de las asignaturas de Bachillerato en modalidad deArtes, la mitad, un $54.1 \%$ de la muestra estaría más de acuerdo en colaborar con la asignatura de Fundamentos del Arte, seguidos de Filosofía (24.6 $\%$ ) y Lengua Castellana y Literatura (13.1\%) (pregunta 13).

\begin{tabular}{|c|c|c|}
\hline & \multicolumn{2}{|c|}{ Representación muestra } \\
\hline & Frecuencia & Porcentaje \\
\hline \multicolumn{3}{|c|}{$\begin{array}{l}\text { Pregunta 9. ¿Dónde crees que puedes aportar tus conocimientos de } \\
\text { música, danza española y bail e flamenco con mayores beneficios? }\end{array}$} \\
\hline Educación Infantil & 7 & 11.5 \\
\hline Educación Primaria & 28 & 45.9 \\
\hline Educación Secundaria & 8 & 13.1 \\
\hline Bachillerato & 3 & 4.9 \\
\hline Universitaria & 15 & 24.6 \\
\hline \multicolumn{3}{|c|}{$\begin{array}{l}\text { Pregunta 10. Si participaras en un proyecto educativo en Educación } \\
\text { Primaria icon qué asignatura estarías más cómodo dentro de las } \\
\text { troncales? }\end{array}$} \\
\hline Ciencias $\mathrm{N}$ atural eza & 11 & 18 \\
\hline Ciencias Sociales & 18 & 29.5 \\
\hline Lenguay L Literatura & 20 & 32.8 \\
\hline Matemáticas & 5 & 8.2 \\
\hline Lengua Extranjera & 7 & 11.5 \\
\hline \multicolumn{3}{|c|}{$\begin{array}{l}\text { Pregunta 11. Si participaras en un proyecto educativo en Educación } \\
\text { Secundaria ¿con qué asignatura estarías más cómodo dentro de las } \\
\text { troncales? }\end{array}$} \\
\hline Biología y Geología & 6 & 9.8 \\
\hline Geografía e Historia & 28 & 45.9 \\
\hline Lengua y Literatura & 21 & 34.4 \\
\hline Matemáticas & 3 & 4.9 \\
\hline Primera Lengua Extranjera & 3 & 4.9 \\
\hline \multicolumn{3}{|c|}{$\begin{array}{l}\text { Pregunta 12. Dentro de las asignaturas específicas o de libre } \\
\text { configuración en Educación Secundaria donde podrías aportar más: }\end{array}$} \\
\hline Educación Física & 37 & 60.7 \\
\hline Filosofía & 8 & 13.1 \\
\hline Música & 16 & 26.2 \\
\hline \multicolumn{3}{|c|}{$\begin{array}{l}\text { Pregunta 13. Si participaras en un proyecto educativo en } \\
\text { Bachillerato en la modalidad de arte con que asignatura estarías más } \\
\text { cómodo dentro de las troncales: }\end{array}$} \\
\hline Filosofía & 15 & 24.6 \\
\hline Fundamentos del Arte & 33 & 54.1 \\
\hline Lengua y Literatura & 8 & 13.1 \\
\hline Lengua Extranjera & 5 & 8.2 \\
\hline
\end{tabular}

Por su parte, un $63.9 \%$ piensa que la danza española y el baile flamenco servirían tanto para aprender otras asignaturas, como para potenciar la vivencia del patrimonio cultural (pregunta 14). Además, un $82 \%$ de los participantes opina que la danza española y el baile fla menco tendrían que estar incluidos en la enseñanza general (pregunta 15). Asimismo, se constata unanimidad en cómo se debería impartir la asignatura de danza española y baile flamenco: un $98.4 \%$ está de acuerdo en que seríavinculando contenidosteóricosy prácticos (pregunta 16). Además, un $85.2 \%$ que opina que un consejo de artistas y pedagogos es el más indicado para diseñar la implementación de la danza en la escuela, descartando a los políticos (pregunta 17).

Planteada la creatividad como otro de los beneficios del aprendizaje de la danza española y el baile flamenco en la escuela, el $100 \%$ de los encuestados opina que se trata de una manera creativa de captar la atención de Iosjóvenes (pregunta 18). En otro orden, un $96.7 \%$ de la muestra entiende que la educación artística impartida en el sistema educativo español no está actualizada (pregunta 19).

Tabla 5

Resultados relacionados con la danza española y el baile flamenco como asignatura

\begin{tabular}{|c|c|c|}
\hline & \multicolumn{2}{|c|}{ Representación muestra } \\
\hline & Frecuencia & Porcentaje \\
\hline \multicolumn{3}{|c|}{$\begin{array}{l}\text { Pregunta 14. La enseñanza de la danza española y el baile flamenco en } \\
\text { la escuela, ¿debería ayudar a aprender otras asignaturas o debería } \\
\text { enseñar se porque ambas tienen valor en sí mismas? }\end{array}$} \\
\hline $\begin{array}{l}\text { Debe real izar se atendiendo a que es un patrimonio cultural y } \\
\text { artístico que todos tenemos derecho a vivenciar }\end{array}$ & 22 & 36.1 \\
\hline $\begin{array}{l}\text { Debe enfocarse para aprender mejor otras materias y } \\
\text { mejorar el rendimiento escolar }\end{array}$ & - & \\
\hline Las dos primeras respuestas son correctas & 39 & 63.9 \\
\hline \multicolumn{3}{|l|}{$\begin{array}{l}\text { Pregunta 15. ¿Crees que en el currículo escolar debería haber una } \\
\text { asignatura específica dedicada a la danza española y el baile flamenco? }\end{array}$} \\
\hline Sí & 50 & 82 \\
\hline No & 6 & 9.8 \\
\hline No lo sé/ no contesta & 5 & 8.2 \\
\hline \multicolumn{3}{|l|}{$\begin{array}{l}\text { Pregunta 16. ¿Q uét tipo de contenidos deberían impartirse en una } \\
\text { asignatura específica dedicada a la danza española a yal baile flamenco? }\end{array}$} \\
\hline Prácticos & 1 & 1.6 \\
\hline Teóricos y prácticos & 60 & 98.4 \\
\hline \multicolumn{3}{|l|}{$\begin{array}{l}\text { Pregunta 17. ¿Q ué colectivo debería diseñar la implementación de la } \\
\text { danza españolay el baile flamenco en el currículo escolar? }\end{array}$} \\
\hline Artistas & 4 & 6.6 \\
\hline Pedagogos & 2 & 3.3 \\
\hline Políticos & 2 & 3.3 \\
\hline Consejo artistas/ pedagogos & 52 & 85.2 \\
\hline No lo sél no contesta & 1 & 1.6 \\
\hline \multicolumn{3}{|l|}{$\begin{array}{l}\text { Pregunta 18. ¿Te parece importante poder usar el arte para captar la } \\
\text { atención de los ióvenes y ayudarles a aprender de manera creativa? }\end{array}$} \\
\hline Sí & 61 & 100 \\
\hline No & - & - \\
\hline \multicolumn{3}{|l|}{$\begin{array}{l}\text { Pregunta 19. ¿Crees que la educación reglada obligatoria está obsoleta } \\
\text { en cuanto a enseñanza de las artes se refiere? }\end{array}$} \\
\hline Sí & 59 & 96.7 \\
\hline No & 1 & 1.6 \\
\hline No lo sél no contesta & 1 & 1.6 \\
\hline
\end{tabular}

Infraestructuras de la escuela para impartir danza y realizar actividades culturales en la educación general

Por otra parte, un $88.5 \%$ de los participantes del estudio piensa que las instalaciones de las aulas se podrían adaptar para realizar actividades de danza (pregunta 20). A pesar de ello, el $47.5 \%$ prefiere un teatro y el $31.1 \%$ el salón de actos del colegio (pregunta 21).

Tabla 6.

Resultados relacionados con las infraestructuras para impartir danza en la escuela

\begin{tabular}{|c|c|c|}
\hline & $\begin{array}{l}\text { Representaci } \\
\text { Frecuencia }\end{array}$ & $\begin{array}{l}\text { ión muestra } \\
\text { Porcentaje }\end{array}$ \\
\hline \multicolumn{3}{|c|}{$\begin{array}{l}\text { Pregunta 20. Crees que el espacio de las clases de colegios e institutos } \\
\text { pueden ser utilizados también para impartir actividades rel acionadas } \\
\text { con la danza española. }\end{array}$} \\
\hline Sí, se podría adaptar & 54 & 88.5 \\
\hline No, no es un espacio adecuado & 7 & 11.5 \\
\hline No lo sél no contesta & - & \\
\hline \multicolumn{3}{|c|}{$\begin{array}{l}\text { Pregunta 21. ¿C uál consideras que es el espacio ideal para enseñar } \\
\text { danza españolay baile flamenco en la escuela? }\end{array}$} \\
\hline Aula música & 5 & 8.2 \\
\hline Polideportivo & 4 & 6.6 \\
\hline Salón de actos & 19 & 31.3 \\
\hline Tablao & 4 & 6.6 \\
\hline Teatro & 29 & 47.5 \\
\hline \multicolumn{3}{|c|}{$\begin{array}{l}\text { Pregunta 22. ¿Consideras que se podrían montar espectáculos } \\
\text { dancísticos como her ramienta alternativa de aprendizaje en la } \\
\text { educación general? }\end{array}$} \\
\hline Sí & 58 & 95.1 \\
\hline No & 1 & 1.6 \\
\hline No lo sé/ no contesta & 2 & 3.3 \\
\hline \multicolumn{3}{|c|}{$\begin{array}{l}\text { Pregunta 23. ¿Pueden ser los espacios de representaciones escénicas de } \\
\text { diversa índole (tablaos, teatros, auditorios, etc.) lugares de aprendizaje } \\
\text { de la enseñanza general obligatoria? }\end{array}$} \\
\hline Sí & 45 & 73.8 \\
\hline No & 7 & 11.5 \\
\hline No lo sél no contesta & 9 & 14.8 \\
\hline
\end{tabular}


Por otra parte, un $95.1 \%$ de los participantes opina que podrían adecuar sus espectáculos para utilizarlos como vía de aprendizaje (pregunta 22). Así también, un 73.8\% considera que los espacios escénicos tradicional es como tablaos, teatros y auditorios podrían adecuar sus programaciones a los fines educativos de la escuela (pregunta 23).

\section{Situación actual sobre la implantación de la danza dentro de la enseñanza general}

Respecto al conocimiento de las leyes de educación y sobre si estas incluyen a los artistas en las aulas, un $95.1 \%$ piensa que el marco regulador no incluye dichas disciplinas (pregunta24), mientras que un $98.4 \%$ opina que sí se debería incluir a los artistas en las aulas (pregunta 25). Como esto no se produce, las asignaturas que tienen una relación con la danza están siendo impartidas por otros profesores, que en la opinión de un $82 \%$ de losencuestados, no poseen suficientesconocimientospara enseñar danza (pregunta 26). Además, un $80.3 \%$ de los participantes cree que podría impartir cursos y seminarios sobre danza para poder formar a los profesores que actualmente imparten actividades relacionadas con la danza en la escuela (pregunta 27). En el caso que tuviera que compartir asignaturas o conocimientos con otros profesores, el $50.8 \%$ de los participantes se siente más cómodo con los de Historia del Arte. Se observa a lo largo de la encuesta la poca afinidad de los artistas

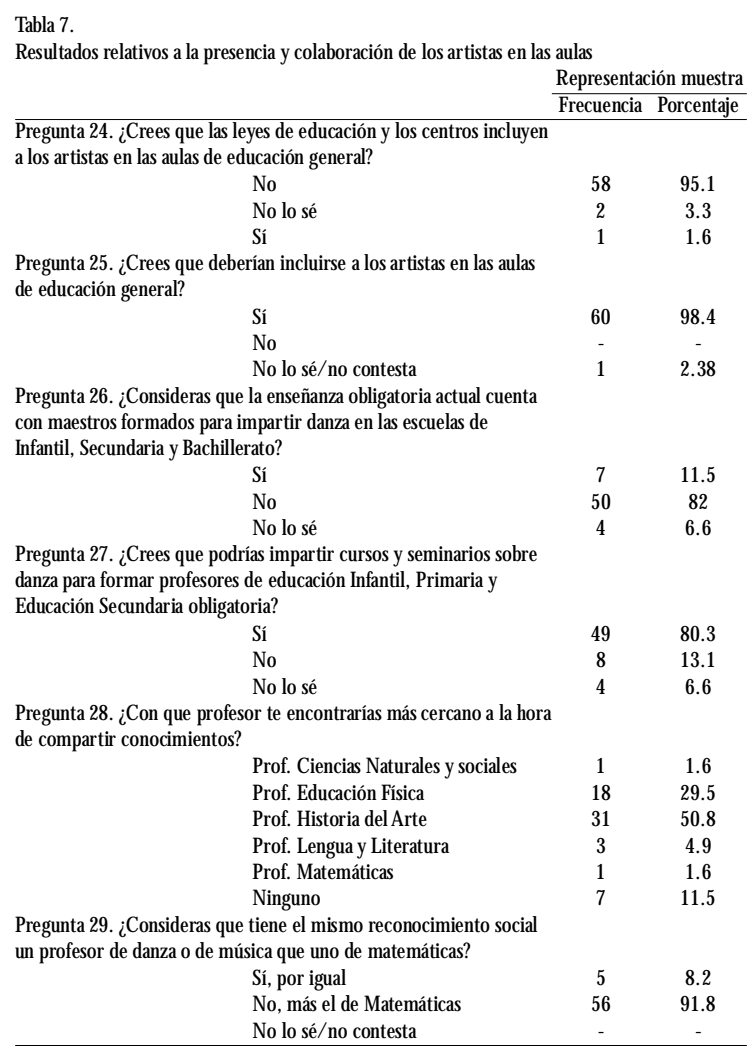

con las M atemáticas, que vuelve a obtener el valor más bajo (pregunta 28). Comparando el reconocimiento social de un profesor de danza y uno de matemáticas, el 91.8\% de los participantes expresa que está más reconocido el profesor de matemáticas, frente a un $8.2 \%$ que piensa que se produce en términos similares (pregunta 29).

La última pregunta se hizo abierta, solicitando a los participantes que agregaran cualquier otra cuestión relacionada con el objeto de estudio, que no hubiera sido abordada previamente (pregunta 30). Los artistas ponen el acento en las implicaciones humanistas de la enseñanza de la danza española y el baile flamenco, considerando su prácticacomo instrumento fundamental para el desarrollo del niño y la configuración de su personalidad. Subrayan otros beneficios como el componente motivacional, la coordinación, la memoria y la concentración. Además, inciden en la orientación no profesional izadora de este tipo de enseñanza, que deberá estar más centrada en incentivar la sensibilización y la relación con el arte. Se propone que se fomente su inclusión en libros de texto y hacen especial hincapié en los contenidos históricos, así como en la aproximación crítica que aborde los límites estéticos, sociopolíticos y filosóficos de la danza española y el baile flamenco.

\section{Discusión y conclusiones}

Los resultados permiten arrojar luz sobre las configuraciones autoperceptivas del rol de losartistas de danza española y baile flamenco en la escuela. Los mismos son alentadores en cuanto al interés que presenta este colectivo en jugar un rol relevante en la educación artística presente y futura de los más jóvenes en contextos no profesionalizadores. M uchos de ellos todavía no se han iniciado en proyectos vinculados a la educación obliga toria, y, pese a ello, un 86.9 \% están dispuestos a negociar sus identidades profesionales (Burnard et al., 2018) e invertir su capital artístico en el futuro de la sociedad, brindando un papel preeminente a las etapas educativas primaria en un $45.9 \%$ y universitaria en un $24.6 \%$. Cabe plantearse si el escaso protagonismo de Secunda ria (13.1\% de los participantes) tiene que ver con las dificultades del trabajo con los adolescentes, pues a la luz de los datos ofrecidos por Zakaras y Lowell (2004) el mayor impacto de la educación artística sobre el consumo cultural de la vida adulta está determinado por su consumo previo entre los 12 y 25 años, coincidentes con la Educación Secundaria y universitaria.

El estudio expone un amplio espectro de beneficios 
derivados de la implementación de la danza española y el baile flamenco en las aulas. El hecho de que un $82 \%$ de la muestra conecte la implementación de la danza española y el baile flamenco en la escuela, con la visión patrimonial del arteautóctono y su incidencia en el campo de la identidad y del sentido de comunidad, parece alentar una nueva orientación curricular hacia esta perspectiva, comprometiendo a la agenda educativa a buscar en la diversidad, multiculturalidad e inclusión promovidas por el arte (Gutiérrez, 2010; Padial-Ruz, Ibáñez-Granados, Fernández \& U bago-Jiménez, 2019; Rodríguez-Q uiles, 2006), una estrategia para mejorar la convivencia en comunidad. A demás de las habilidades físicas, cognitivas y psicosociales adquiridas a través de la danza, los artistas subrayan los efectos humanizadores de las artes. Por su parte, el $100 \%$ de la muestra considera a la danza fundamental para el desarrollo de la creatividad de los educandos, en línea con Hefferon y Ollis (2006) y M éndez-Giménez (2020).

Las leyes educativas españolas, por su parte, siguen esperando un abordaje serio para su actualización en esta materia. Son asignaturas pendientes, tanto el fomento de la presencia de los artistas en las aulas - estos lo apoyan en un $98.4 \%$ - , como la incorporación de la danza española y el baile flamenco a las aulas, que, sal vo por contadas iniciativas autonómicas, subsiste en forma de proyectos particulares. Los exiguos contenidos de danza del currículo continúan siendo impartidos por docentes poco formados para ello (Cuéllar \& Pestano, 2013; García, 2002; Troya \& Cuéllar, 2013), así lo reflejan los encuestados en un $82 \%$. En un $80.3 \%$, los artistas están dispuestos a colaborar en la formación en danza de estos docentes y en la incorporación transversal de la misma en sus asignaturas impartiendo cursos y seminarios que son escasos y serían beneficiosos (Peña de Hornos \& Vicente, 2019). Q ueda por concretar las estrategias de habilitación para que los titulados superiores y graduados universitarios en danza puedan impartir estos contenidos en la escuela y explorar las vías de colaboración de los artistas profesionales de élite con los docentes general istas, especialistas y profesores-artistas.

Un $98.4 \%$ de los artistas se inclina hacia un enfoque esencialista y desde él considera importante la incorporación de contenidos teóricos y prácticos de la danza española y el baile flamenco como una asignatura específica del currículo escolar por su valor intrínseco. Tampoco descartan la perspectiva contextualista de la educación artística y en este sentido, se muestran más proclives a colaborar con profesores y asignaturas de corte humanístico, además de Educación Física donde en un $60.7 \%$ se sienten cercanos a esta asignatura, mostrándose más distanciados de Matemáticas - única mente un $1.6 \%$ de la muestra pese a que en otros contextos geográficos es una de las áreas que suele conectar bien con ladanza( (M atthews, 2012; Palmer, 2010). Además, un $85.2 \%$ de los artistas que han participado del estudio consideran que la garantía de calidad de la inclusión de la danza en la escuela pasa por monitorizarla mediante un consejo independiente de la política, conformado por artistas y pedagogos.

En cuanto a las infraestructuras necesarias para impartir danza en la escuela, pese a que en un $88.5 \%$ se consideran aptas las instalaciones de los colegios, deberán tomarse en cuenta los equipamientos cultural es públicoscomo centrosculturales, teatrosy auditorios, para fomentar el arte en la comunidad. Tal como señalába mos, el necesario trabajo cooperativo de la escuela con los artistas suele encontrar trabas burocráticas de las administraciones para ceder estos espacios y adecuar su programación a los fines educativos (Algar $\&$ Algar, 2015).

En otro orden, el presente estudio presenta limita ciones, entre las que destacan (i) la muestra y (ii) la herramienta de recogida de datos. Respecto a la primera - tratándose de un muestreo no probabilístico-, no existe equiprobabilidad en la selección de posibles participantes, determinando que las conclusiones obtenidas no puedan ser extrapoladas a toda la población, por lo que resulta recomendable desarrollar en el futuro un estudio con muestreo probabilístico. En relación a la herramienta de recogida de datos, es importante destacar queloscuestionariosautocumplimentados, pese a resultar herramientas probadas de recogida de datos en el entorno cuantitativo y poseer la ventaja inestima ble de su bajo coste y facilidad de difusión para llegar al mayor número posible de participantes, presentan el inconveniente de que las respuestas pueden verse influenciadas por estados anímicos y emocionales puntuales de los sujetos.

Finalmente, este trabajo se presenta como una aproximación inicial a la implicación de los artistas de la danza española y el baile flamenco para determinar su rol en la mejora del sistema educativo español y el alcance de su potencial contribución a una educación artística de calidad, factores determinantes para la adquisición de competencias necesarias de los ciudadanos del siglo XXI y la adaptación a los cambios de paradigma a los que estamos asistiendo a todos los niveles. Tal como se ha señalado, al tratarse de un estudio descriptivo de 
un universo concreto, las conclusiones no son generalizables. Pese a ello, este trabajo resulta crucial para establecer líneas prospectivas que permitan un mayor alcance de estos presupuestos. A partir del mismo, se propone que para investigaciones futuras se rea lice un abordaje más concreto desde diferentes extractos y variables, así como una indagación de las formas de colaboración con profesores y asignaturas de la educación general y artística, estableciendo las vías de acceso de los docentes-artistas y de los artistas en colaboración. Por su parte, un mapeo cualitativo a partir de entrevistas semiestructuradas cuyos registros sean procesados mediante un análisis sociológico del discurso, permitiría indagar en profundidad en las configuraciones subjetivas de los artistas y docentes implicados en los procesos de enseñanza aprendizaje de la danza en la escuela, cuyos resultados podrían enlazar de forma más estrecha, enriquecedora y efectiva a la escena con las aulas.

\section{Referencias}

Algar, M.A. , \&Algar, M. L. (2015). Ladanzaespañolaen el ámbito escolar. En Chinchilla, J.L., y Díaz, O. (Coord.). Danza, educación einvestigación, (55-63). Málaga: EdicionesAljibe.

Alvira, F. (2011). Cuadernos metodológicos. La encuesta: una pers pectiva general metodológica, (35), 15-31. Madrid: CIS.

Anderson, B. (1993). Comunidades imaginadas. Reflexiones sobre el origen y la difusión de nacionalismo. Méico: Fondo de Cultura Económica.

Anttila, E., \& Svendler, C. (2019). Danceand the quality of life at schools: A Nordic affilizion. In Dance and the quality of life, (73), (327-345). Springer, Cham. https:/ / doi.org/ 10.1007/ 978-3-319-95699-2 10

Burnard, P., Holliday, C., Jasilek, S. \& Nikolova, A. (2018). Artists and arts-based method usein higher eduction:A livinginquiry of an academic programme in a faculty of education. In Artsbased Methods and Organizational Learning, (291-325). Cham: Palgrave Macmillan. https:/ / doi.org/ 10.1007/ 978-3-31963808-9 13

Chappell, K., Rolfe, L., Jobbins, V., \& Craft, A. (2011). Close Encounters:D ancePartnersfor Creativity. Stoke-on-Trent:Trentham Books Ltd. http:// dx. doi.org/ 10.1080/ 15290824.2012 .757816

Conservatorio Superior de Danza «M aría de Ávila». Recuperado de: https:/ / www. csdma.es/ conservatorio/ departamentos/ educativacomunitaria

Craft,A. (2005). Creativity in school stensionsand dil lemmas.Abingdon: Routledge. http:/ / dx. doi.org/ 10.4324/ 9780203357965

Craft,A. (2011). Creativity and Education Futures: Learning in a digital age. Stoke-on-Trent: Trentham Books Ltd. Recuperado de: http:/ / dx. doi.org/ 10.1080/ 01411926.2011.605110

Cuéllar, M. J., \& Pestano, M. A. (2013). Formación del Profesora- do en Expresión Corporal: planes de estudio y Educación Física. Retos. N uevastendenciasen educación física, deportey recreación, (24), 123-128. Murcia: Federación Españolade Docentes de Educación Física. Recuperado de: http:/ / www. retos.org/

Day, M. D. (1986). Artist-teacher: A problematic model for art education. Journal of aesthetic Education, 20(4), 38-42. Recuperado de: http:/ / www.jstor.org/ stable/ 3332595 http:/ / dx. doi.org/ 10.2307/ 3332595

DeLas Heras, R., \& Calderón, D. (2018). El zapateado flamenco en lasaulas. unarevisión alalegislación. Arteduca, (19), 52-61. http:/ / dx. doi.org/ 10.6035/ artseduca. 2018.19.3

DeLasHeras, R., \& García, D. (2019). Estudio delosprocesosde enseñanza aprendizajedel flamenco atravésdeentrevistas. La visión de las mujeres. Revista Electrónica LEEME, (43), 50-73. Recuperado de: https: / / ojs. uv. es/ index. php/ LEEME http:/ / dx.doi.org/ 10.7203/ leeme. 43.13587

Díaz, A. (2012). Intervención en el aula a través deladanza folclórica. Ensayos, (27), 101-108. Recuperado de: https: / / www. revista. uclm.es/ index. php/ ensayos

Eisner, W. E. (2008). Art and knowledge. En Knowles, J. G., \& Cole, A. L. (Eds). H andbook of the Arts in Qualitative Research. Perspectives, Methodol ogies, Examples, and Issues. Sage Publications.

García, H. (2002). La danza en la escuela y la formación de los profesores. Contextos educativos. Revisa de educación, (5), 173184. https:/ / doi.org/ 10.18172/ con.511

Gardner, H. (1998). M entescreativas: U na anatomía de la creatividad. Barcelona: EdicionesPaidós Ibérica, S.A.

Giménez, F. A. (2012). Proyecto flamencas una experiencia didáctico-flamenca en la ESO. Revista de Investigación sobre Flamenco «La Madrugá», (7). Recuperado de: https:/ / revistas. um.es/ flamenco/ article/ view/ 163451

Grande, J. (2005). Flamenco en el Aula. Sevilla: ConsejeríadeEduca ción de laJ untadeAndalucía.

Gutiérrez, R. (2010). ¿Seaprendeflamenco en el sistemaeducativo andaluz? Revista del nvestigación sobreFlamenco "La M adrugá», (3). Recuperado de: https:/ / revistas. um.es/ flamenco/ article/ view/ 113231

$\mathrm{Hall}$, J. (2010). M kingart, teachingart, learningart: Exploringthe concept of theartistteacher. International Journal of Art \& D esign Education, 29(2), 103-110. https:/ / doi.org/ 10.1111/ j.1476-8070.2010.01636.x

Hefferon, K. M., \& Ollis, S. (2006). 'Justclicks': an interpretative phenomenological analysis of professional dancers' experience of flow. Research in Dance Education, 7(2), 141-159. https:/ / doi.org/ 10.1080/ 14647890601029527

Holguín, S. (2013). «Vergüenzay ludibrio de lasciudadesmodernas): losnacionalistascatalanesanteel flamenco en laBarcelona de 1900-1936. Hispania, 73(244), 439-468. https:/ / doi.org/10.3989/ hispania. 2013.013

Horowitz, R. (2003). Executivesummary: N ational danceinstitute's in-school education programs eval uation. Recuperado de: http:/ / www. national dance.org/ NDI Awards Recognition. pdf Jiménez, M. D. (2017). El flamenco en el aula no es algo novedoso e CO: Revista digital de educación y formación del pro- 
fesorado, (14), 27-34. Recuperado de: http:// revistæeco.cepcordoba. org/ index.php/ 2017/ 01/ 17/ el-fla menco-en-el-aula-no-es-algo-novedoso/ amp/

Klimenko, 0. (2008). Lacreatividad como un desafío paralaeducación del siglo XXI. Educación y educadores, 11(2), 191-210. Recuperado de: http:/ / www.scielo.org co/ pdf/ eded/ v11n2/ v1ln2a12.pdf

Laban, R. (1978). Danza Educativa M oderna. BuenosAires: Editorial Paidós.

López, F. (2018). Lacategoríade «danzaespañola» en M ariemma: Mis caminos a través de la danza. En Mariemma y su tiempo, (195-209). Madrid: INAEM/ CDMD/ CSDMA.

López, M. (2004). Introducción al flamenco en el currículum escolar. Sevilla: Akal-U niversidad Internacional deAndalucía.

López, N. , López,A., \&Vicente, G. (2020). Ladanzaen Educación Física: análisis de los currículos autonómicos españoles de Educación Primaria (Dance in Physical Education: analysis of the Spanish regional curricula of Primary Education). Retos: nuevas tendencias en educación física, deporte y recreación, (38), 517-522. Recuperado de: https: / recyt. fecyt.es/ index. php/ retos/ article/ view/ 77413 https:/ / doi.org/ 10.47197/ retos. v38i38. 77413

Mariemma(1997). Tratado de Danza Española. Mariemma. Miscaminosa través de la danza. Madrid: Fundación Autor.

Matos-Duarte, M., Smith, E., \& Muñoz, A. (2020). Danzas folclóricas: unaformade aprender y educar desdelaperspectiva sociocultural. Retos. nuevas tendencias en educación física, deportey recreación, (38), 739-744. Recuperado de: https:/ / recyt. fecyt. es/ index. php/ retos/ article/ view/ 73725/ 49508

Matthews, C. (2012). Maths as storytelling: Maths is beautiful. En Aboriginal andTorresStrait Isander education:An introduction for theteaching profession, (94-112). Cambridge University Press. https:/ / doi.org/ 10.1017/ CB0 9781316134481.007

M éndez-Giménez, A. (2020). Resultadosacadémicos, cognitivos yfísicosdedosestrategiasparaintegrar movimiento en el aula: clases activas y descansos activos. SPORT TK Revista EuroAmericana De Ciencias De Deporte, 9(1), 63-74. https:/ / doi.org/ 10.6018/ sportk. 412531

Muñoz, J. R. (1996). Estrategias didácticas para el tratamiento del flamenco en el aula. Comunicación presentada en LosCantesy el flamenco deAlmería: I Congreso Provincia: Baños de Sierra Alhamilla, 101-112. Almería: Instituto de Estudios Almerienses.

O rtiz, M. M. (2015). Ladanzaenlaenseñanzaobligatoria Ladanza española en el ámbito escolar. En Danza, Investigación y Educación: experiencias interdisciplinares con música, literatura y teatro, (55-63). Ediciones Aljibe.

Padial-Ruz, R., Ibáñez-Granados, D., Fernández, M., \& U bagoJiménez, J. L. (2019). Proyecto de baile flamenco: desar rollo motrizy emocional en educación infantil. Retos, nuevastendenciasen educación física, deportey recreación, (35), 396-401. Recuperado de: https:/ / recyt.fecyt. es/ index. php/ retos/ article/ view

Palmer,A. (2010).'Let'sdance!'Theorisingalternativemathematical practices in early childhood teacher education. Contemporary
Issues in Early Childhood, 11(2), (130-143). https:/ / doi.org/ 10.2304/ ciec. 2010.11.2.130.

Peña de Hornos, V. J., \& Vicente, G. (2019). Danza en educación infantil: opinión delos docentes. Retos. nuevastendenciasen educación física, deporte y recreación, (36), 239-244.

Robinson, J. (1999). El niño y la danza. Barcelona: 0 ctaedro.

Rodríguez-Q uiles, J. A. (2006). Flamenco, pedagogía de la diferencia y formación inicial del profesorado de música Revista Electrónica deLEEME, (18). Recuperado de: https:/ / ojs uv.es/ index. php/ LEEME/ article/ view/ 9762

Rubio, J. A. (2016). Dimensionesy papel de laeducación artística en laspolíticas educativasy culturales en la Españademocrática. En Treinta años de políticas culturales en España: Participación cultural, gobernanza teritorial e industrias culturales, (321-339). Universidad deValencia.

Runco, M. A. (1989). The creativity of children's art. Child Study Journal, 19(3), 177-189.

Salazar, M . (2018). Flamenco en el aula. Educación musical: Recursos para el cambio metodológico. Procompal Publicaciones.

The National Society for Education in Art and Design. NSEAD. Recuperado de: https: / www.nsead. org

Thornton, A. (2005). The artist teacher as reflective practitioner. International Journal of Art\& Design Education, 24(2), 166-174. https:// doi.org/ 10.1111/ j.14768070.2005.00437.x

Touriñán, J.M. (2011). Claves para aproximarse a la educación artística en el sistema educativo: educación «por» las artes y educación «para» un arte. ESE. Estudiossobreeducación, (21), 6181. Servicio de Publicaciones de la Universidad de Navarra. Recuperado de: http:/ / hdl. handle. net/ 10171/ 22549

Trigo, E., Rodríguez, I. M., \& M oreno, P. (2019). . M iraquéalegría! Promoviendo el conocimiento de la culturaandaluza en Educación Secundaria O bligatoria: una propuestadidáctica. Álabe 19. https:/ / doi.org/ 10.15645/ alabe2019.19.7

Troya,Y., \& Cuéllar, M.J. (2013). Formación docentey tratamiento de la danza en Canarias: evaluación desde la Educación Física. Retos. Nuevastendenciasen educación física, deportey recreación, (24), 165-170. Recuperado de: http:/ / www. retos.org/ numero_24/ 165-170.pdf

Unesco. (2006). Hoja de ruta paralaeducación artística. Conferencia mundial sobre la educación artística: Construir capacidades creativas para el siglo XXI. Recuperado de: https:/ / www.unesco.org/ new/ fileadmin/ multimedia/ hq/ clt/ clt/ pdf/ arts_edu_roadmap_es

Unesco. (2010). El Flamenco Patrimonio Cultural Inmaterial. Recuperado de: https:/ / ich.unesco.org/ es/ RL/ el-flamenco-00363

Utrilla, J. (2007). El flamenco seaprende.Teoría didáctica para la ense ñanza del flamenco. Córdoba: EdicionesToro Mítico, S. L.

W inner, E., Goldstein, T., \&Vicent-Lancrin, S. (2014). iEl artepor $d$ arte? La influencia dela educación artística. OECD Publishing https:/ / doi.org/ 10.1787/ 9789264224902-es

Zakaras, L., \& Lowell, J. (2008). Cultivating demand for thearts:Arts learning, artsengagement, and statearts policy. Rand Corporation. https:/ / doi. org/ 10.7249/ MG640 